\title{
Reactivity descriptors and electron density analysis for ligand chemistry: A case study of 2,2'-bipyridine and its analogues
}

\author{
BHAKTI S KULKARNI, ${ }^{1}$ AKHILESH TANWAR ${ }^{2}$ and SOURAV PAL ${ }^{1, *}$ \\ ${ }^{1}$ Physical Chemistry Division, National Chemical Laboratory, Pune 411008 \\ ${ }^{2}$ Scientific and Engineering Computing Group, Centre for Development of Advanced Computing, \\ Pune 411007 \\ e-mail: s.pal@ncl.res.in
}

MS received 12 July 2007; accepted 13 September 2007

\begin{abstract}
In this paper, we study the reactivity of diimines like 2,2'-bipyridine and its analogues using reactivity descriptors. We discuss evaluation of local descriptors using relaxed as well as frozen approximation and characterize the $\sigma / \pi$ acceptance/donor characteristics of the above ligands. The intermolecular reactivity sequence for the same systems is examined by the global and local philicity index. In addition, electron density analysis has been carried out to highlight the possible strengths of interaction of the bipyridine and its analogues with metal ions.
\end{abstract}

Keywords. 2,2'-Bipyridine; reactivity descriptors; DFT.

\section{Introduction}

The prediction of changes in reactivity and selectivity at particular atom centers is an important concept related to the activation (de-activation) at that site. Site activation (de-activation) may be promoted in several ways, including solvation effects, substitution of a particular functional group, the presence of a reagent or a catalyst or in general by any external source of perturbation. Such prediction has been one of the objectives of chemical reactivity indices.

In recent years, density functional theory (DFT) has emerged as a powerful tool through which chemical concepts such as reactivity, selectivity, and reaction path of a system have been studied. ${ }^{1-5}$ The concepts of chemical potential $(\mu)$, electronegativity, and hardness $(\eta),{ }^{6-8}$ collectively known as global reactivity descriptors, have systematized the study in this area. The principle of maximum hardness(PMH), ${ }^{9}$ relating the relative stability of a system to a larger value of hardness, has been tested using semi-empirical as well as $a b$ initio quantum chemical techniques. ${ }^{10}$ Local reactivity descriptors, such as Fukui function (FF) and local softness, relating changes in electron density to the number of electrons and chemical potential, respectively, have been used to determine the site-

\footnotetext{
*For correspondence
}

reactivity of a system. ${ }^{11}$ Electrophilic and nucleophilic FFs have been used as indicators of reactivity to nucleophilic and electrophilic reagents, respectively. ${ }^{12}$ Roy et $a l^{13}$ proposed that relative electrophilicity and relative nucleophilicity, based on the ratio of electrophilic and nucleophilic FFs and its inverse, are more reliable descriptors to locate the preferable sites for electrophilic and nucleophilic attack respectively within a molecule. Parr and co-workers have defined a new concept of global philicity ${ }^{14 a}$ using which Chattaraj and co-workers have defined local philicity indices. ${ }^{14 \mathrm{~b}}$ The importance of charges and different frontier-controlled descriptors in description of electrostatic interactions in ligand chemistry has been highlighted in the past. ${ }^{14 \mathrm{c}}$ Recently, Tanwar et al proposed two reactivity descriptors viz. normalized Fukui function (NFF) and bond deformation kernel $(B D K)^{14 d}$ for comparative studies on the systems with varying number of atoms. A local version of the hard-soft-acid-base (HSAB) principle, proposed by Gazquez and Mendez, ${ }^{15}$ and pursued by Pal and co-workers ${ }^{16}$, as well as, Geerlings and co-workers ${ }^{17}$ to a variety of chemical situations, is suited to semiquantitative description of interaction energy.

In this report, we present a study of the reactivity descriptors in explaining the donor-acceptor character of the 2,2'-bipyridine and its important analogues 3-(2-pyridinyl)-1, 2, 4-triazine and 3-(2- 
pyrazinyl)-1,2,4-triazine, generated by substitution of $-\mathrm{CH}$ group by $\mathrm{N}$-atoms in the bipyridine ring. We observe that this class of systems presents an example where cation is to be exercised in the calculation of condensed FF. We discuss two different schemes of calculations of the Fukui functions and related descriptors, one using 'relaxed orbital' and the other 'frozen orbital' approach. Depending on the symmetry of the orbitals involved during complexation, the ligands are generally classified as $\sigma$-donor, $\pi$-donor and $\pi$-acceptor ligands. ${ }^{18} 2,2^{\prime}$-bipyridine is both a $\sigma$-donor and $\pi$-acceptor ligand. ${ }^{19}$ We have also used electron density to highlight the possible strengths of interaction of the bipyridine and its analogues with metal ions. There has been significant interest in bipyridine and polypyridine complexes of heavy metal ions due to their inherently interesting and unusual excited state properties and the potential utility of this class of compounds in solar energy conversion schemes. ${ }^{20-26}$ The ability of bipyridines to form ionic and neutral guest compounds makes them useful precursors in supra-molecular chemistry. ${ }^{19,27}$ Moreover, self-organization of bipyridine structures and metal cation often leads to the formation of helicates, which are supra-molecular complexes formed between pre-organized receptors and metal ions. ${ }^{28-30}$ In addition to the areas mentioned above, bipyridine units are also significant in catalytic studies. ${ }^{31,32}$ Polypyridine complexes constitute an important class of redox mediators in biosensors. ${ }^{33}$ Studies in this area focus on design and control for the selective preparation of complicated organized chemical structures. Simple concepts based on density descriptors can facilitate controlled selective preparation of the above complexes by identification of reactivity of the different sites of the systems.

The paper has been organized as follows. In $\S 2$, we describe theoretical background leading to definitions of Fukui functions, philicity, local philicity indices, relative electrophilicity and nucleophilicity. Section 3 provides the computational details and the methodology used for calculations. In $\S 4$, we focus the results and discussion of the various calculations for the model systems.

\section{Theoretical background}

According to Hohenberg-Kohn (HK) theorem, the ground state energy of atom or molecule is written as a function of electron density $(\rho):{ }^{34 a}$

$$
E[\rho]=\int \rho(r) v(r) d r+F_{H K}[\rho],
$$

with

$$
F_{H K}[\rho]=T[\rho]+V_{e e}[\rho],
$$

where $v(r)$, defined in the (1), is the external potential and $F_{H K}$ is the universal Hohenberg-Kohn functional, which comprises of electronic kinetic energy functional $(T[\rho])$ and electron-electron interaction functional $\left(V_{e e}[\rho]\right)$.

The first and second partial derivatives of $E[\rho]$, with respect to the number of electrons $N$ under the constant external potential $v(r)$, are defined as the chemical potential $(\mu)$ and the global hardness $\eta$ of the system, respectively. ${ }^{6-8}$ The global softness $(S)$ is the half inverse of the hardness. The global descriptor of hardness has been known as an indicator of the overall stability of the system. ${ }^{9}$

It has been customary to use finite difference approximation for the computation of $\mu$ and $\eta:^{3 a}$

$$
\begin{aligned}
& \mu=-\frac{(I+A)}{2} \\
& \eta=\frac{1}{2}(I-A),
\end{aligned}
$$

where $I$ and $A$ are the first vertical ionization energy and electron affinity of chemical species respectively. Under the Koopmanns' approximation, ${ }^{34 \mathrm{~b}}$ working equations of chemical potential and hardness turn out as follows,

$$
\begin{aligned}
& \mu=\frac{\varepsilon_{\mathrm{HOMO}}+\varepsilon_{\mathrm{LUMO}}}{2} \\
& \eta=\frac{\varepsilon_{\mathrm{HOMO}}-\varepsilon_{\mathrm{LUMO}}}{2}
\end{aligned}
$$

where $\varepsilon_{\text {HOMO }}$ and $\varepsilon_{\text {LUMO }}$ are the orbital energies of highest occupied molecular orbital (HOMO) and lowest unoccupied molecular orbital (LUMO) of the $N$ electron system respectively.

The site-selectivity of a chemical system, cannot, however, be studied using the global descriptors of reactivity. For this, appropriate local descriptors need to be defined. An appropriate definition of local softness $s(r)$ is given by ${ }^{11}$ 


$$
s(r)=\left(\frac{\partial \rho(r)}{\partial N}\right)_{v(r)}\left(\frac{\partial N}{\partial \mu}\right)_{v(r)}=f(r) S
$$

Such that,

$$
\int s(r) d(r)=S
$$

where $f(r)$ is defined as the Fukui function. ${ }^{11}$ It is obvious that local softness contains the same information as the Fukui function, ${ }^{35}$ in addition, information to the softness of the whole molecule. FF can be interpreted either as the change of the electron density $\rho(r)$ at each point $r$ when the total number of electrons is changed or as the sensitivity of a system chemical potential to an external perturbation at a particular point $r$.

$$
f(r)=\left(\frac{\partial \rho(r)}{\partial N}\right)_{v(r)}=\left(\frac{\partial \mu}{\partial v(r)}\right)_{N} .
$$

The latter point of view, by far the most prominent in the literature, faces the $\mathrm{N}$-discontinuity problem of atoms and molecules, ${ }^{36}$ leading to the introduction ${ }^{11 a}$ of both right- and left-hand-side derivatives, both to be considered at a given number of electrons, $N=N_{0}$ :

$$
f^{+}(r)=\left(\frac{\partial \rho(r)}{\partial N}\right)_{v(r)}^{+}
$$

The above equation (10) describes nucleophilic attack provoking increase of an electron in the system. Similarly,

$$
f^{-}(r)=\left(\frac{\partial \rho(r)}{\partial N}\right)_{v(r)}^{-}
$$

describes electrophilic attack provoking decrease of an electron in the system.

Although, in principle, the electron density of neutral or $N_{0}$-electron system contains all information needed for the evaluation of the Fukui function, most studies in the literature have been carried out using the finite difference method, approximating,

$$
f^{+}(r) \approx \rho_{N_{0}+1}(r)-\rho_{N_{0}}(r),
$$

and

$$
f^{-}(r) \approx \rho_{N_{0}}(r)-\rho_{N_{0-1}}(r)
$$

A third function describing radical attack, $f^{0}(r)$, is then obtained as the arithmetic average of $f^{+}(r)$ and $f^{-}(r)$.

Since the derivative of (9) is not known exactly, there are various different strategies to calculate, in an approximate way, the Fukui function. Two different finite-difference approaches can be followed. Separate calculations of $\left(N_{0}+1\right)$ and $\left(N_{0}-1\right)$ electrons imply relaxation of orbitals from the neutral system. This approach has been known as the 'relaxed orbital' approach. The practical difficulty of the FF, especially of $f^{+}(r)$, using this approach suffers the technical difficulties of computing the density of anionic system at the same level of accuracy as the neutral system. At this stage it is difficult to get the correlation effect. A manner of taking into account, in an approximate way, the orbital relaxation effects doing a single point calculation on the neutral system and avoiding the complexity of the anionic species has been presented and implemented in the literature including the work of present authors. On the contrary, assuming that the shape of molecular orbitals does not change when a small amount of charge is added or subtracted, Yang et al calculated the derivative of (9) as, governing electrophilic attack,

$$
f^{-}(\vec{r}) \approx \rho_{\mathrm{HOMO}}(\vec{r})
$$

governing nucleophilic attack,

$$
f^{+}(\vec{r}) \approx \rho_{\mathrm{LUMO}}(\vec{r})
$$

and governing radical attack,

$$
f^{0}(\vec{r}) \approx \frac{1}{2}\left[\rho_{\text {НОмО }}(\vec{r})+\rho_{\mathrm{LUMO}}(\vec{r})\right]
$$

Known as the 'frozen orbital' approximation, where $\mathrm{d} \rho$ is same as $\mathrm{d} \rho_{\text {valance. }}{ }^{11 \mathrm{a}}$ However, limitations of the frozen orbital approximation has been analysed, within the conceptual DFT framework. ${ }^{11 \mathrm{c}}$ Apart from these two approximations completely different route for the evaluation of the FF can also be pursued. However, approximations other than 'relaxed orbital' and 'frozen orbital' indirectly involve the quality of functionals entering in $F$, and hence are less reliable.

For determining site-selectivity or site reactivity one usually calculates what are called atom-condensed Fukui functions, first introduced by Yang et al based on idea of integrating the Fukui function over atomic regions, ${ }^{12}$ similar to the procedure followed 
in population analysis technique. ${ }^{37}$ Combined with finite difference approximation, this yields working equations of the type,

$$
\begin{aligned}
& f_{A}^{+}=q_{A, N_{0}+1}-q_{A, N_{0}}, \\
& f_{A}^{-}=q_{A, N_{0}}-q_{A, N_{0-1}},
\end{aligned}
$$

where, $q_{A, N_{0}}$ denotes the electronic population of atom $\mathrm{A}$ of reference system. In $a b$ initio calculations these numbers are obtained mostly by a Mulliken and Lowdin population ${ }^{38}$ analysis. Under frozen orbital approximation atom-condensed Fukui functions will be nothing but the respective atomic population of HOMO or LUMO orbitals.

In addition to the FF, other reactivity descriptors based on FF have also been used. For example, relative electrophilicity and relative nucleophilicity, defined as $\left(f^{+} / f^{-}\right)$and $\left(f^{-} / f^{+}\right)$respectively, ${ }^{28 a}$ are greatly used to explain intra and inter molecular reactivity. Parr et $a l^{14 a}$ proposed a global philicity $(W)$ as $\mu^{2} / 2 \eta$. Using this, Chattaraj et al ${ }^{14 b}$ proposed the existence of local philicity index $w(r)$ as $W f(r)$ such that $w(r)$ integrates to global $W$. The atom-condensed philicity, $w_{A}^{a}$ in the definition is given by

$$
w_{A}^{a}=W f_{A}^{a}, \forall a=+,-, 0 .
$$

Earlier Krishnamurty et $a l^{39 a}$ proposed 'group softness' to describe intermolecular reactivity trends in carbonyl compounds and organic acids. They defined group softness as the sum of softness of atoms present in an appropriately defined group. Based on this concept, Chattaraj and co-workers ${ }^{39 c}$ proposed the 'group philicity' as the summed condensed-philicity of atoms present in the group.

$$
w_{g}=\sum_{k=1}^{n} w_{k}
$$

where $n$ is the number of atoms bonded to the reactive atom, $w_{k}$ is the atom-condensed philicity of the atom $k$, and $w_{g}$ is the group philicity.

These reactivity descriptors have been used extensively for the study of site selectivity of atoms in a molecule..$^{13,39-42}$ In this paper these quantities will be computed and its validity is tested in predicting the intramolecular and intermolecular reactivity trends.

\section{Computational details and methodology}

DFT calculations were performed to test the validity of the different approaches (frozen orbital versus relaxed orbital approach) using the GAMESS ${ }^{43}$ system of programs. The ground state geometries of 2,2'bipyridine ligand and its substituted analogues namely 3-(2-pyridinyl)-1,2,4-triazine and 3-(2-pyrazinyl)1,2,4-triazine were completely optimized with split valence basis set 6-31G $(d, p)$ along with B3LYP hybrid functional. The electronic populations on the atoms for these systems were obtained from Lowdin population analysis.

\section{Results and discussion}

Figure 1 presents the optimized structures of $2,2^{\prime}-$ bipyridine, 3-(2-pyridinyl)-1,2,4-triazine and 3-(2pyrazinyl)-1,2,4-triazine. The interaction at a molecular level depends on both electron donating and accepting character of the system, which in turn is dependent on the substitution.

\subsection{Global reactivity}

Table 1 presents the ionization energies, electron affinities and global reactivity of the ligands. The substitution of $-\mathrm{CH}$ groups by $\mathrm{N}$ atoms at bipyridine ring do not change the structural parameters widely. However, due to re-distribution of atomic charges within the molecule, there is a significant change in the magnitude of global properties. With the increase of $N$ atoms in the ring, electron affinities increases linearly, however, the change in ionization potential is not prominent. Similar conclusion can be drawn from the chemical potential and hardness parameters reported in table 1. Based on principle of maximum hardness (MHP), the molecular stability has been extensively studied employing chemical potential and chemical hardness. According to this principle the minimum energy system has maximum chemical hardness value. Hence, MHP is a qualitative tool to study the stability of the system. The stability decreases as more number of $-\mathrm{CH}$ groups are substituted with $N$ atoms. However, the global softness increases with the $N$ substitution, predicting higher polarizability, hence reactivity of the systems. To analyse the change in electrophilic power of the systems, the molecular electrophilic power has been calculated. In general, the substituted $N$ atoms enhance the electrophilic power irrespective 
Table 1. Ionization energies, electron affinities and global reactivity.

\begin{tabular}{lccc}
\hline Properties (a.u.) & 2,2'-Bipyridine & Pyridinyltriazine & Pyrazinyltriazine \\
\hline Ionization energy & 0.2321 & $0 \cdot 2259$ & 0.2337 \\
Electron affinity & 0.0543 & $0 \cdot 0713$ & 0.0808 \\
Chemical potential & $-0 \cdot 1432$ & $-0 \cdot 1486$ & -0.1572 \\
Chemical hardness & 0.0889 & $0 \cdot 0773$ & 0.0764 \\
Softness & 5.6242 & $6 \cdot 4683$ & $6 \cdot 5445$ \\
Philicity & 0.1153 & $0 \cdot 1428$ & 0.1616
\end{tabular}
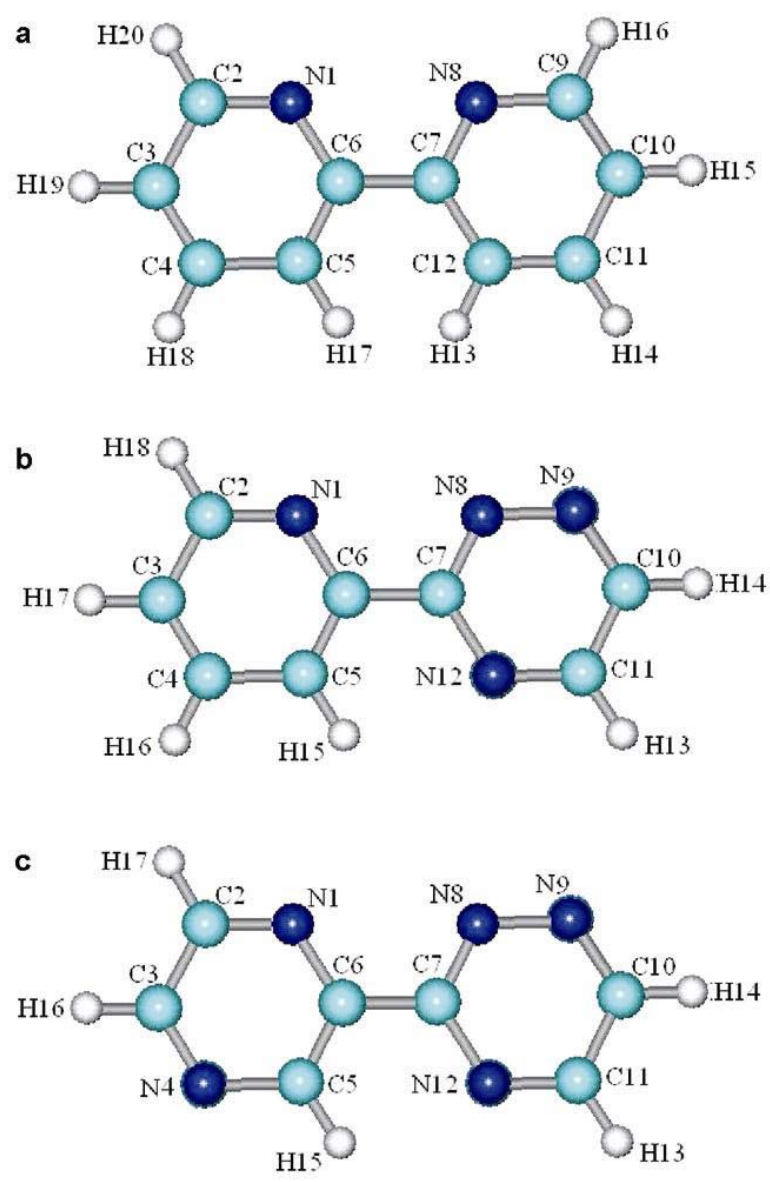

Figure 1. (a) 2,2'-Bipyridine (b) 3-(2-pyridinyl)-1,2,4triazine (c) 3-(2-pyrazinyl)-1,2,4-triazine. Expected metal binding sites are $\mathrm{N}(1)$ and $\mathrm{N}(8)$, metal approach in the plane of molecule.

of the nature and position of the substitution. For pyridinyl triazine this substitution is only at one pyridine ring, whereas for pyrazinyl triazine it is at both rings. As we go from bipyridine to triazines, the values of these properties are increased.

\subsection{Local reactivity: Relaxed versus Frozen ap- proximation}

$2,2^{\prime}$-bipyridine is one of the critical case studied here for understanding the prediction of observed experimental results. This is a typical case where relaxed orbitals could not predict the correct acceptance/donor character. Table 2 highlights the failure of the atom-condensed Fukui functions in explaining electrophilic and nucleophilic attacks using relaxed orbitals. The Nitrogen atoms, carrying lone pair of electrons are expected to be prominent $\sigma$-donors, which, is not observed by looking at the $f^{-}$values on $\mathrm{N}$ atoms. However, it instead predicts the electron deficient ring carbon atoms to be a competent nucleophile. On the contrary, the same ring carbon atoms along with nitrogen atoms are shown to be equally good electrophile, which goes against the expected reactivity. Here this discrepancy lies with calculation of nucleophilic Fukui functions. The HOMOs of $N_{0}-1$ and $N_{0}$ state of 2,2'-bipyridine are shown in figure $2 \mathrm{a}$ and $2 \mathrm{~b}$ respectively. This should be noted that the HOMO of neutral 2,2'-bipyridine is of $\sigma$-type while that of the cationic 2,2'-bipyridine is $\pi$ type. As can be seen from the (9), the definition of Fukui function involves derivative of $\rho(r)$ with respect to infinitesimally small number of electrons $(\delta N)$. This addition or deletion of $\delta N$ number does not allow the relaxation of orbitals. Table 3 presents the orbital energies of HOMO and LUMO of both $\sigma$ and $\pi$ type orbitals. HOMO of $\pi$-type and $\sigma$ type are nearly degenerate in case of $2,2^{\prime}$-bipyridine. The removal of one electron from neutral 2,2'-bipyridine causes too much relaxation and the symmetry of (HOMO-1) orbital and HOMO orbital of the ion changes with respect to the neutral system. As a result of this, it appears that the electron is removed from HOMO-1 ( $\pi$-type) instead of the HOMO ( $\sigma$ type). Hence, it is more prudent to calculate FF us- 
Table 2. Atom condensed Fukui functions of the systems using relaxed orbitals.

\begin{tabular}{|c|c|c|c|c|c|c|}
\hline \multirow[b]{2}{*}{ Atom number } & \multicolumn{2}{|c|}{ 2,2'-Bipyridine } & \multicolumn{2}{|c|}{ Pyridinyltriazine } & \multicolumn{2}{|c|}{ Pyrazinyltriazine } \\
\hline & $f^{+}$ & $f^{-}$ & $f^{+}$ & $f^{-}$ & $f^{+}$ & $f^{-}$ \\
\hline 1 & 0.0538 & 0.0485 & 0.0468 & 0.0959 & 0.0763 & 0.0846 \\
\hline 2 & 0.0096 & 0.0346 & 0.0322 & 0.0432 & 0.0298 & 0.0488 \\
\hline 3 & 0.0585 & 0.0543 & 0.0992 & 0.0518 & $0 \cdot 1134$ & $0 \cdot 0555$ \\
\hline 4 & 0.0345 & 0.0296 & 0.0379 & 0.0438 & 0.0617 & 0.0950 \\
\hline 5 & 0.0005 & 0.0318 & 0.0453 & 0.0368 & 0.0372 & $0 \cdot 0407$ \\
\hline 6 & $0 \cdot 0211$ & 0.0137 & 0.0332 & 0.0057 & 0.0470 & 0.0150 \\
\hline 7 & $0 \cdot 0211$ & 0.0137 & 0.0832 & 0.0563 & 0.0811 & $0 \cdot 0494$ \\
\hline 8 & 0.0538 & 0.0485 & 0.0336 & 0.1121 & 0.0383 & 0.0971 \\
\hline 9 & 0.0096 & 0.0346 & $0 \cdot 1088$ & 0.1233 & 0.0832 & $0 \cdot 1151$ \\
\hline 10 & 0.0585 & 0.0543 & $0 \cdot 1176$ & 0.0663 & $0 \cdot 1219$ & 0.0639 \\
\hline 11 & 0.0345 & 0.0296 & 0.0359 & 0.0645 & 0.0333 & 0.0598 \\
\hline 12 & 0.0005 & 0.0318 & 0.1144 & 0.0827 & 0.0881 & 0.0733 \\
\hline
\end{tabular}

Figures in bold letters indicates $\mathrm{N}$ atom's $\mathrm{FF}$ values where as others indicate $\mathrm{C}$ atom's FF values
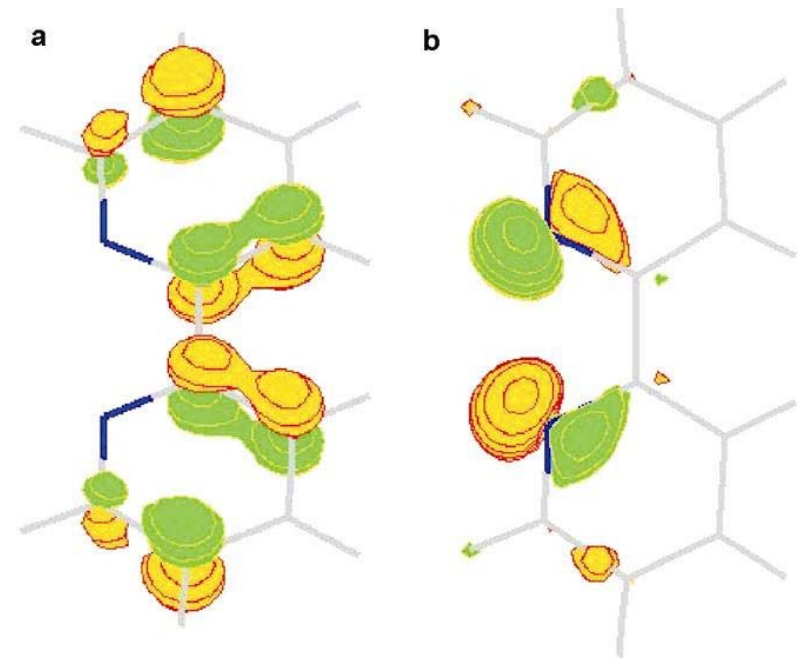

Figure 2. HOMO density plots (a) $N_{0}-1$ state of 2,2'bipyridine (b) $N_{0}$ state of 2,2'-bipyridine.

ing addition or deletion of one electron, but using orbitals which are frozen (frozen orbital approximation). On removal and addition of an electron to the ground state of a molecular system, only the frontier molecular orbitals (FMO) will undergo the changes. The frozen orbital approach is thus more reliable as it avoids undue relaxation and will be used for further calculations.

\subsection{Intra and intermolecular reactivity sequence}

The electrophilic and nucleophilic FFs calculated under frozen orbital approximation are presented in table 4. Though, the analogues of 2,2'-bipyridine, considered here using relaxed orbitals (see table 2) were indeed predicting the $\sigma$-donation from nitrogen and $\pi$-acceptance from ring carbon atoms for pyridinyl triazine and pyrazinyl triazine, these effects were only moderate, as indicated by the small values. Electrophilicity or nucleophilicity is highlighted more with the use of frozen orbitals. All the three molecules show the highest nucleophillic FF at $N_{8}$. 2,2'-bipyridine shows highest and equal nucleophillic FFs for $N_{1}$ and $N_{8}$ where as electrophilic FFs for $\mathrm{C}_{3}-\mathrm{C}_{10}, \mathrm{C}_{6}-\mathrm{C}_{7}$. The results obtained so reflects symmetry of 2,2'-bipyridine confirming the equal probability for $\sigma$-donation and $\pi$-acceptance. In Pyridinyl triazine, $\mathrm{C}_{9}-\mathrm{H}_{16}$ and $\mathrm{C}_{12}-\mathrm{H}_{13}$ of $2,2^{\prime}-$ bipyridine are substituted by electron withdrawing $N$ atoms. With these additional $N$ atoms not only symmetrical geometry is lost but also reactivity is affected. The major donation is seen at $N_{8}$ and $N_{9}$ whereas $N_{12}$ partially contributes. No donation is observed through $N_{1}$ in pyridine ring. In this system, when we look at the $f^{+}$values of $N_{9}$ and $N_{12}$, it is higher than $f^{-}$values thereby proving to be better $\pi$-acceptor sites within the molecule. In addition, $\mathrm{C}_{10}$ and $\mathrm{C}_{11}$ of the triazine ring affirm nucleophilic attack. Similarly, for pyrazinyl triazine, the lone pair donation is through $N_{8}$ and $N_{9}$. The $\mathrm{C}_{7}$ and $\mathrm{C}_{10}$ have higher electrophilic FFs, but $N_{9}$ and $N_{12}$ of pyrazinyl ring do not depict as strong $\pi$-acceptance character as pyridinyl triazine. The additional $N$ atom substituted for $\mathrm{C}_{4}-\mathrm{H}_{18}$ of the 2,2'-bipyridine increases the acceptance at $\mathrm{C}_{3}$ and $\mathrm{C}_{6}$ of the pyrazinyl ring. 
Table 3. Orbital energies (a.u.).

\begin{tabular}{lccrcc}
\hline \multirow{2}{*}{ Orbital symmetry } & \multicolumn{2}{c}{ HOMO } & & \multicolumn{2}{c}{ LUMO } \\
\cline { 2 - 3 } \cline { 5 - 6 } & $\sigma$ & $\pi$ & & $\sigma$ & $\pi$ \\
\hline 2,2'-Bipyridine & -0.2317 & -0.2324 & & 0.0882 & -0.0459 \\
Pyridinyltriazine & -0.2259 & -0.2545 & & 0.0985 & -0.0713 \\
Pyrazinyltriazine & -0.2337 & -0.2675 & & 0.1036 & -0.0808 \\
\hline
\end{tabular}

Table 4. Atom condensed Fukui functions of the systems using frozen orbital approximation.

\begin{tabular}{|c|c|c|c|c|c|c|}
\hline \multirow[b]{2}{*}{ Atom number } & \multicolumn{2}{|c|}{ 2,2'-Bipyridine } & \multicolumn{2}{|c|}{ Pyridinyltriazine } & \multicolumn{2}{|c|}{ Pyrazinyltriazine } \\
\hline & $f^{+}$ & $f^{-}$ & $f^{+}$ & $f^{-}$ & $f^{+}$ & $f^{-}$ \\
\hline 1 & $0 \cdot 1039$ & $0 \cdot 2572$ & 0.0160 & 0.0956 & 0.0689 & 0.0986 \\
\hline 2 & 0.0049 & 0.0495 & 0.0081 & 0.0100 & 0.0072 & 0.0164 \\
\hline 3 & $0 \cdot 1482$ & 0.0523 & 0.0366 & 0.0076 & 0.0984 & $0 \cdot 0154$ \\
\hline 4 & 0.0530 & $0 \cdot 0092$ & 0.0024 & $0 \cdot 0010$ & 0.0219 & 0.0551 \\
\hline 5 & $0 \cdot 0463$ & $0 \cdot 0185$ & 0.0332 & $0 \cdot 0108$ & $0 \cdot 0381$ & $0 \cdot 0186$ \\
\hline 6 & $0 \cdot 1383$ & 0.0533 & 0.0264 & $0 \cdot 0091$ & 0.0734 & $0 \cdot 0156$ \\
\hline 7 & $0 \cdot 1383$ & 0.0533 & $0 \cdot 0822$ & 0.0636 & $0 \cdot 1339$ & 0.0599 \\
\hline 8 & $0 \cdot 1039$ & 0.2571 & 0.0505 & $0 \cdot 3157$ & 0.0099 & 0.2648 \\
\hline 9 & 0.0049 & 0.0495 & 0.2575 & 0.2546 & 0.1529 & 0.2319 \\
\hline 10 & $0 \cdot 1482$ & $0 \cdot 0523$ & $0 \cdot 1132$ & $0 \cdot 0592$ & $0 \cdot 1568$ & $0 \cdot 0524$ \\
\hline 11 & 0.0531 & 0.0092 & 0.1009 & 0.0276 & 0.0689 & $0 \cdot 0986$ \\
\hline 12 & $0 \cdot 0463$ & $0 \cdot 0185$ & $0 \cdot 2673$ & $0 \cdot 1202$ & 0.0072 & 0.0164 \\
\hline
\end{tabular}

Figures in bold letters indicates $\mathrm{N}$ atom's FF values where as others indicate $\mathrm{C}$ atom's FF values

Table 5 focuses on the atom-condensed philicity and group philicity values of bipyridine and its analogues. The philicity index is used to compare the 3 molecules. In case of 2,2'-bipyridine, $N_{1}$ and $N_{8}$ shows similar probability for the electrophilic attack whereas in the analogues only triazine ring is active with highest nuclephilic FF at $N_{8}$ and no contribution from $N_{1}$ of the adjacent ring. This suggests the loss of chelation in the two analogues. The experimentally observed metal approach distances (see table 6) supports these site selectivity calculations, where metal is closer to the $N_{8}$ of the triazine rings. Also from the local philicity, we observe that $N_{8}$ of pyridinyl triazine shows higher probability for electrophillic attack where as $N_{9}, N_{12}$ shows higher probability for nucleophillic attack compared to $2,2^{\prime}$-bipyridine and pyrazinyl triazine. The global philicity for $2,2^{\prime}$-bipyridine is 0.1153 and increases to $0 \cdot 1428$ and $0 \cdot 1616$ for pyridinyl triazine to pyrazinyl triazine respectively. We have also calculated the group philicity (both donation and acceptance character) of atoms in the triazine ring (atoms numbering 7 to 12 ) of pyridinyl triazine and pyrazinyl triazine and observe that the reactivity of this ring is lower in pyrazinyl triazine, although overall reactivity of pyrazinyl triazine is higher. This is due to the substitution of one $\mathrm{CH}$ group by $N$ atom in the adjacent ring in the pyrazinyl compound. This shows higher donation and acceptance at triazine ring of pyridinyl triazine than the pyrazinyl triazine.

In order to discuss the electrophilicity or nucleophilicity of the systems from the perspective of the type of orbital involved for the attack, Geerlings and co-workers ${ }^{44}$ separated the $\sigma$ and $\pi$ molecular orbitals to calculate the Fukui functions. The idea of separateing the $\sigma$ and $\pi$ molecular orbitals is extremely important to study the supra-molecular formation involving such class of systems. However, within the framework of frozen orbital approximation, $\sigma-\pi$ separation is more trivial and the following relations will hold: 
Table 5. Atom condensed philicity and group philicity of the systems using frozen orbital approximation.

\begin{tabular}{|c|c|c|c|c|c|c|}
\hline \multirow[b]{2}{*}{ Atom number } & \multicolumn{2}{|c|}{ 2,2'-Bipyridine } & \multicolumn{2}{|c|}{ Pyridinyltriazine } & \multicolumn{2}{|c|}{ Pyrazinyltriazine } \\
\hline & $w^{+}$ & $w^{-}$ & $w^{+}$ & $w^{-}$ & $w^{+}$ & $w^{-}$ \\
\hline 1 & 0.0119 & 0.0296 & 0.0022 & 0.0136 & $0 \cdot 0111$ & 0.0159 \\
\hline 2 & $0 \cdot 0005$ & $0 \cdot 0057$ & $0 \cdot 0011$ & $0 \cdot 0014$ & $0 \cdot 0011$ & 0.0026 \\
\hline 3 & $0 \cdot 0170$ & $0 \cdot 0060$ & 0.0052 & $0 \cdot 0010$ & $0 \cdot 0159$ & $0 \cdot 0024$ \\
\hline 4 & $0 \cdot 0061$ & $0 \cdot 0010$ & $0 \cdot 0003$ & $0 \cdot 0001$ & 0.0035 & 0.0089 \\
\hline 5 & 0.0053 & 0.0021 & $0 \cdot 0047$ & $0 \cdot 0015$ & $0 \cdot 0061$ & 0.0030 \\
\hline 6 & 0.0159 & $0 \cdot 0061$ & 0.0037 & $0 \cdot 0012$ & $0 \cdot 0118$ & 0.0025 \\
\hline$\Sigma$ & 0.0570 & 0.0507 & $0 \cdot 0175$ & $0 \cdot 0191$ & $0 \cdot 0497$ & 0.0355 \\
\hline 7 & 0.0159 & 0.0061 & 0.0117 & 0.0090 & 0.0216 & 0.0096 \\
\hline 8 & 0.0119 & 0.0296 & 0.0072 & 0.0450 & $0 \cdot 0016$ & $0 \cdot 0427$ \\
\hline 9 & $0 \cdot 0005$ & 0.0057 & 0.0367 & 0.0363 & 0.0247 & 0.0374 \\
\hline 10 & $0 \cdot 0170$ & $0 \cdot 0063$ & $0 \cdot 0161$ & $0 \cdot 0084$ & $0 \cdot 0253$ & $0 \cdot 0084$ \\
\hline 11 & $0 \cdot 0061$ & $0 \cdot 0010$ & $0 \cdot 0144$ & 0.0039 & $0 \cdot 0111$ & 0.0159 \\
\hline 12 & 0.0053 & 0.0021 & 0.0381 & 0.0171 & 0.0011 & 0.0026 \\
\hline$\Sigma$ & 0.0570 & 0.0507 & $0 \cdot 1244$ & $0 \cdot 1200$ & $0 \cdot 0855$ & $0 \cdot 1169$ \\
\hline
\end{tabular}

Figures in bold letters indicates $\mathrm{N}$ atom's philicity values where as others indicate $\mathrm{C}$ atom's philicity values.

Table 6. Comparative density values of 2,2'-bipyridine, pyridinyl triazine, pyrazinyl triazine.

\begin{tabular}{lccccc}
\hline \multicolumn{2}{l}{ Distance from $N$ atoms } & & \multicolumn{3}{c}{ Density* $10^{-4}$ (a.u.) } \\
\cline { 1 - 2 } \cline { 5 - 6 }$N(1)$ & $N(8)$ & & $2,2^{\prime}$-Bipyridine & Pyridinyltriazine & Pyrazinyltriazine \\
\hline 2.257 & $2 \cdot 139$ & & $49 \cdot 70$ & $32 \cdot 83$ & 27.55 \\
$2 \cdot 262$ & $2 \cdot 104$ & & 54.82 & $38 \cdot 14$ & 31.99 \\
2.296 & $2 \cdot 124$ & & 51.78 & 35.58 & 29.70 \\
\hline
\end{tabular}

$$
\begin{aligned}
& f^{-} \approx f_{x}^{-} \quad \forall\{H O M O \in x \mid x=\sigma, \pi\} \\
& f^{+} \approx f_{x}^{+} \quad \forall\{L U M O \in x \mid x=\sigma, \pi\}
\end{aligned}
$$

hence values presented in table 4 for $f^{-}$and $f^{+}$are nothing but $f_{\sigma}^{-}$and $f_{\pi}^{+}$, respectively. The high value of $f_{\sigma}^{-}$on $N$ atoms using HOMO indicates high reactivity for an electrophilic attack in the molecular plane of ring, hence proving $\sigma$-donation through $N$ atom. Similarly, $f_{\pi}^{+}$obtain through LUMO indicates that it is very likely for a nucleophile to attack in the plane perpendicular to molecular plane of the ring. The stereographs of HOMO and LUMO of all three ligands are shown in figure 3 . The density plots show that HOMO is of $\sigma$-symmetry and LUMO is of $\pi$-symmetry for each system. As observed from the plots, HOMO contribution mainly comes from the $\sigma$-orbitals centered on the nitrogen atoms and LUMO contributions from the $P_{z}$ orbitals of $\mathrm{C}$ atoms in case of 2,2'-bipyridine whereas additional triazine
$\mathrm{N}$ atoms also contributes in case of its analogues. All the three ligands are therefore of the same class i.e. $\sigma$-donor and $\pi$-acceptor.

\subsection{Electron density analysis}

Table 6 presents the electron density values calculated at different distances (experimentally observed for metal approach) for the bipyridine and its analogues. It may, however, be noted that experiments have been carried out in solution phase where as our calculations are done on the gas phase. As our main interest here is to count the $\sigma$-donation character of three molecules, we focus on the $\rho(r)$ numbers. The electron density values for bipyridine analogues are lower than the bipyridine. This drop is primarily due to the substitution of $-\mathrm{CH}$ groups by $\mathrm{N}$ atoms. 2,2'bipyridine being both $\sigma$-donor and $\pi$-acceptor, the lone pair on nitrogen atom forms a $\sigma$-bond with the central metal atom while the aromatic system takes 
part in back bonding. 2,2'-bipyridine having two $N$ donor atoms, separated by two carbon atoms, form a five member ring, which is the most stable structure. The diimine part of the bipyridine delocalizes the
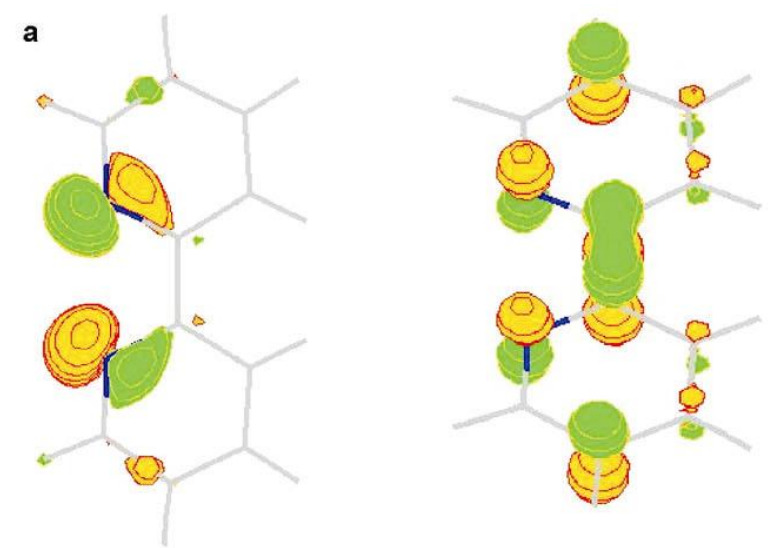

b
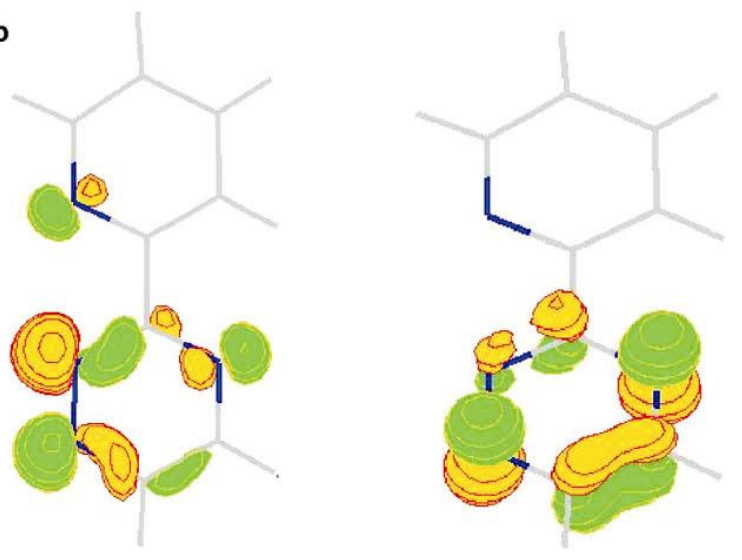

C

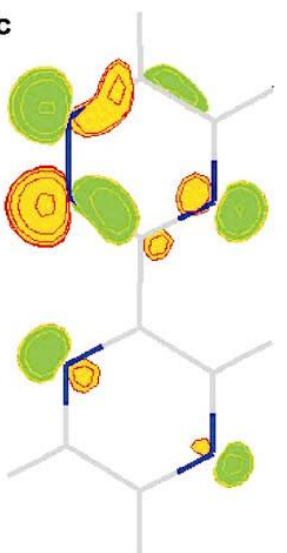

HOMO electrons in the chelate ring. ${ }^{19}$ This chelation is no longer effective for pyridinyl triazine and pyrazinyl triazine as additional $N$ atoms act as electron withdrawing agent and thus reducing electron density at diimine nitrogen.

\section{Conclusion}

Two approximations to calculate Fukui functions have been discussed. The significance of frozen orbital approximation compared to relaxed orbital approximation is highlighted with the illustrative example. Atom-condensed Fukui functions within the FOA, clearly predicts the intra-molecular reactivity, electron rich $N$ atoms being dominant nucleophile where as electron deficient $\mathrm{C}$ atoms as electrophiles. Further, the inter-molecular reactivity index 'philicity' interprets that as the more number of $-\mathrm{CH}$ groups are substituted by $N$ atoms, only triazine ring gets activated with the highest donor character at $N_{8}$ atom, where as $N_{1}$ has no contribution, there by reducing the chelation at diimine nitrogen atoms. This is also supported by our electron density calculations. Electron density analysis shows that the electron density around the bonding distances of $\mathrm{N}$-atoms are higher in bipyridine ring, compared to the density of the corresponding atoms in pyridinyl triazine and pyrazinyl triazine. The calculation of group philicity of triazine ring shows lower donation or acceptance character of the ring in pyrazinyl compound than the pyridinyl compound, though latter has overall lower reactivity. The idea of calculating descriptors using $\sigma$ and $\pi$ molecular orbital separation is impelling to explain the formation of supra-molecular assemblies through sigma-pi complexation. All the three molecules are of $\sigma$-donor and $\pi$-acceptor type. 2,2'bipyridine being stronger $\sigma$-donor and its analogues being better $\pi$-acceptor.

\section{Acknowledgment}

We thank Prof. Animesh Chakravorty, Department of Inorganic Chemistry, IACS, Kolkata for introducing to us the importance of reactivity of bi-pyridine systems, providing us the inspiration and valuable suggestions for this work as well as his reading of the manuscript. One of the authors (BSK) acknowledges Department of Science and Technology, New Delhi, India for financial assistance.
Figure 3. HOMO and LUMO density plots (a) 2,2'bipyridine (b) 3-(2-pyridinyl)-1,2,4-triazine (c) 3-(2pyrazinyl)-1,2,4-triazine. 


\section{References}

1. Gazquez J L 1997 J. Phys. Chem. 1018967

2. Nguyen L T, Le T N, De Proft F, Chandra A K, Langenaeker W, Nguyen M T and Geerlings P $1999 \mathrm{~J}$. Am. Chem. Soc. 1215992

3. (a) Parr R G and Yang W 1989 Density-functional theory of atoms and molecules (New York: Oxford University Press); (b) Geerlings P, De Proft F, Langenaeker W 1999 Adv. Quantum Chem. 33303

4. (a) Chandra A K and Nguyen M T 1998 J. Phys. Chem. A102 6181; (b) Chandra A K, Michalak, Nguyen M T and Nalewajski R F 1998 J. Phys. Chem. A102 10182

5. (a) De Proft F, Amira S, Choho K and Geerlings $\mathrm{P}$ 1994 J. Phys. Chem. 98 5227; (b) Langenaekar W, De Decker M, Geerlings P and Raeymaekars P 1990 J. Mol. Struct. (Theochem.) 207115

6. Pearson R G 1963 J. Am. Chem. Soc. 853533

7. Sen K D 1993 Chemical hardness (structure and bonding) (Berlin: Springer-Verlag) vol 80

8. (a) Parr R G and Pearson R G $1983 \mathrm{~J}$. Am. Chem. Soc. 105 7512; (b) Parr R G, Donnelly R A, Levy M and Palke W E 1978 J. Chem. Phys. 68 3801; (c) Pearson R G 1985 J. Am. Chem. Soc. 1076801

9. (a) Parr R G and Chattaraj P K $1991 \mathrm{~J}$. Am. Chem. Soc. 113 1854; (b) Pearson R G 1987 J. Chem. Edu. 64561

10. (a) Pearson R G and Palke W E 1992 J. Phys. Chem. 96 3283. (b) Datta D 1992 J. Phys. Chem. 96 2409; (c) Pal S, Vaval N and Roy R K 1993 J. Phys. Chem. 974404

11. (a) Parr R G and Yang W 1984 J. Am. Chem. Soc. 106 4049; (b) Yang Y and Parr R G 1985 Proc. Natl. Acad. Sci. USA 821 6723; (c) Garza J, Vargas R, Cedillo A, Galván M and Chattaraj P K 2006 Theor. Chem. Acc. 115257

12. Yang W and Mortier W 1986 J. Am. Chem. Soc. 108 5708

13. (a) Roy R K, Krishnamuthy S, Geerlings P and Pal S 1998 J. Phys. Chem. A102 3746; (b) Roy R K, Pal S and Hirao K 1999 J. Chem. Phys. 1108236

14. (a) Parr R G, Szentpaly L V and Liu S 1999 J. Am.Chem. Soc. 121 1922; (b) Chattaraj P K, Maiti B and Sarkar U 2003 J. Phys. Chem. A107 4973; (c) Chattaraj P K 2001 J. Phys. Chem. A105 511; (d) Tanwar A, Bagchi B and Pal S 2006 J. Chem. Phys. 125214304

15. Gazguez J L and Mendez F 1994 J. Phys. Chem. 98 4591

16. (a) Pal S and Chandrakumar K R S $2000 \mathrm{~J}$. Am. Chem. Soc. 122 4145; (b) Chandrakumar K R S and Pal S 2002 J. Phys. Chem. A106 5737 (c) Chandrakumar K R S and Pal S 2001 J. Phys. Chem. A105 4541

17. (a) De Proft, F and Geerlings P 2001 Chem. Rev. 1011451 (b) Geerlings P, De Proft F and Langenaeker W 2003 Chem. Rev. 103 1793; (c) Mendez F, Tamariz J and Geerlings P 1993 J. Phys. Chem. A97 4059
18. (a) Cotton F A and Wilkinson G 1998 Advanced inorganic chemistry (New York: John Wiley) pp. 3583; (b) Spessard G O and Miessler G L 1997 Organometallic chemistry (New Jersey: Prentice-Hall) pp. $48-137$

19. Vögtle F 1991 Supramolecular chemistry an introduction (Chichester: John Wiley) pp. 9-26.

20. Hegg M J, Kroner R and Deutsch E 1985 Acta. Cryst. C41 684

21. Chakravorty A 2005 Eur. J. Inorg. Chem. 4863; (b) Das S and Chakravorty A 2006 Eur. J. Inorg. Chem. 2285

22. Ghosh P, Pramanik A, Bag N and Chakravorty A 1992 J. Chem. Soc., Dalton Trans. 1883

23. DeArmond M K and Cardin C M 1981 Chem. Rev. 36 325

24. (a) Kitamura N, Kim H B, Kawaniski Y, Obata R and Tazuke S 1986 J. Phys. Chem. 90 1488; (b) Meyer T 1983 J. Prog. Inorg. Chem. 30389

25. Chang Y J, Orman L K, Anderson D R, Yabe T and Hopkins J B 1987 J. Chem. Phys. 873249

26. Denti G, Campagna S, Sabatino L, Serroni S, Ciano $\mathrm{M}$ and Balzani V 1990 Inorg. Chem. 294750

27. Ziessel R and Lehn J M 1990 Helv. Chim. Acta 73 1149

28. Rodriguez-Ubis J C, Alpha B, Plancherel D and Lehn J M 1984 Helv. Chim. Acta 672264

29. Bilyk A and Harding M M 1994 J. Chem. Soc. Dalton Trans. 77

30. Piguet C, Bernardinelli G and Hopfgartner G 1997 Chem. Rev. 972005

31. Haasnoot J G, Hinrichs W, Weir O and Vos J G 1986 Inorg. Chem. 254140

32. Collomb, Dunand, Sauthier M N, Deronizer A, Ziessel R 1994 Inorg. Chem. 332961

33. Zakeeruddin S M, Fraser D M, Nazeeruddin Md K and Grätzel M 1992 J. Electroanal. Chem. 337253

34. (a) Hohenberg P and Kohn W 1964 Phys. Rev. B136 864; (b) Koopmans T 1934 Physica 1104

35. Nalewajski R F and Parr R G 1982 J. Chem. Phys. 77 399

36. (a) Perdew J P, Parr R G, Levy M and Balduz J L 1982 Phys. Rev. Lett. 49 1691; (b) Zang Y and Yang W 2000 Theor. Chem. Acc. 103346

37. For a review, see: Bachrach S M, Lip Kowitz K B and Boyd D B 1995 Reviews in computational chemistry (New York: VCH), vol. V, p. 171.

38. (a) Lowdin P O 1953 J. Chem. Phys. 21 374; (b) Lowdin P O 1950 J. Chem. Phys. 18 365; (c) Mulliken R S 1955 J. Chem. Phys. 231833

39. (a) Krishnamurthy S and Pal S 2000 J. Phys. Chem. A104 7639; (b) Krishnamurthy S, Roy R K, Vetrival R, Iwata S and Pal S 1997 J. Phys. Chem. A101 7253; (c) Parthasarathi R, Padmanabhan J, Elango M, Subramanian V and Chattaraj P K 2004 Chem. Phys. Lett. 394225

40. (a) Russo N, Toscano M, Grand A and Mineva T 2000 J. Phys. Chem. A104 4017; (b) Ghosh S K 1990 Chem. Phys. Lett. 17277 ; (c) Perez P, Toro-Labbe A and Contreras R 2000 J. Phys. Chem. A104 5882 ; (d) Perez P, Zapta-Torres G, Para-Mouchet J and Contre- 
ras R 1999 Int. J. Quantum. Chem. 74 387; (e) Perez P, Toro-Labbe A, Aizman A and Contreras R $2002 \mathrm{~J}$. Org. Chem. 674747

41. (a) Damoun S, Van de woude G, Mendez F and Geerlings P 1997 J. Phys. Chem. A101 886; (b) Mendez F, Romero M de L, De Proft F and Geerlings P 1998 J. Org. Chem. 635774

42. Chatterjee A, jwasaki T and Takeo E 1999 J. Phys. Chem. A103 2489
43. Schmidt M W, Baldrige K K, Boatz J A, Elbert S T, Gordon M S, Jensen J H, Koseki S, Matsunga $\mathrm{N}$, Nguyen $\mathrm{K} \mathrm{A}$, Su S, Windus T L, Dupuis M and Montgometry J A 1993 J. Comput. Chem. 14 1347

44. (a) Langenaeker W, Demel K and Geerlings P 1991 J. Mol. Struct. (Theochem.) 234 329; (b) Langenaeker W, Demel K and Geerlings P 1992 J. Mol. Struct. (Theochem.) 259317 